\title{
3D Craniofacial Morphometric Analysis of Young Subjects with Marfan Syndrome: A Preliminary Report
}

\author{
Claudia DOLCI ${ }^{1}$, Valentina PUCCIARELLI ${ }^{1}$, Marina CODARI ${ }^{1}$, Daniele M. GIBELLI ${ }^{1}$, \\ Susan MARELLI ${ }^{2}$, Giuliana TRIFIRÒ ${ }^{3}$, Alessandro PINI' ${ }^{2}$, Chiarella SFORZA ${ }^{1 *}$ \\ ${ }^{1}$ Dipartimento di Scienze Biomediche per la Salute, Università degli Studi di Milano, Milano, Italy; \\ ${ }^{2}$ Centro Malattie Rare, MarfanClinic, A.O. Luigi Sacco, Milano, Italy; \\ ${ }^{3}$ U.O. Pediatria, A.O. Salvini, Rho (Milano), Italy
}

DOI: 10.15221/15.054 http://dx.doi.org/10.15221/15.054

\begin{abstract}
Marfan syndrome (MFS) is a rare autosomic dominant disease of connective tissues mostly due to mutations in the fibrillin 1 gene. Clinical manifestations of MFS include a variety of signs and symptoms, mainly affecting the heart, blood vessels, bones, joints and eyes, and comprising craniofacial alterations. At present, diagnosis of MFS is largely based on clinical signs and family history. However, it could may be difficult, as its manifestations vary greatly and they are not always present right away. Since a life-threatening complication of MFS is aortic dissection, an early diagnosis of the disorder is essential. We aim to better describe the face of patients with MFS, identifying new quantitative morphological features which could facilitate the early diagnosis of the disease. In the current preliminary study, a group of young subjects with MFS was investigated. Three-dimensional facial images of 3 girls and 8 boys aged 5-15 years were collected by stereophotogrammetry. From the coordinates of 50 anatomical facial landmarks, linear distances and angles were measured; $z$ score values were calculated through the comparison with data obtained from 556 control subjects matched for gender, age, and ethnicity. All subjects with MFS showed a longer face than controls, mainly due to an increased middle third (mean $z$ score $=1.7$ ). They also showed a longer mandibular body (mean $z$ score $=1.4$ ) with a shorter ramus (mean $z$ score $=-1.4$ ) and a greater facial divergence (mean $z$ score = 2.2). The assessment of facial features of subjects with MFS pointed out some morphometric characteristics that had never been reported in literature, alongside with other well known alterations, and suggests the usefulness of a three-dimensional quantitative approach for the recognition of facial phenotypic features of the syndrome. Nevertheless, they need to be confirmed extending the study on more patients.
\end{abstract}

Keywords: Stereophotogrammetry, face, Marfan syndrome (MFS)

\section{Introduction}

Marfan syndrome (MFS, OMIM \#154700) is an autosomic dominant disease of connective tissues due to mutations in the fibrillin 1 gene (FBN1) in more than $95 \%$ of cases, but also to mutations in the transforming growth factor beta receptor 1 and 2 genes (TGF- 31 , TGF- $\beta 2$ ) or in other still unknown genes. The resulting dysregulation of the TGF- $\beta$ signalling affects structural integrity of extracellular matrix. The syndrome is rare, with a prevalence estimated at 1 per 5,000 individuals in the general population, without any racial or gender predilection. Clinical manifestations of MFS reflect the damage to the connective tissues; they include a variety of signs and symptoms, mainly affecting the heart, blood vessels, bones, joints and eyes, and comprising alterations of the craniofacial district [1-3].

At present, diagnosis of MFS is based mainly on clinical signs and familiar history, according to international criteria defined in 2010 (Ghent's criteria) [4]. However, establishing a diagnosis of MFS may be difficult, as its phenotypic expression can vary greatly and the features of the disorder are not always present right away. Moreover, genetic testing for mutations in FBN1 and other genes can help in the diagnosis of MFS but it could be not conclusive.

The most serious clinical and life-threatening complications of MFS result from pathologic changes in the cardiovascular system. In particular the aorta can weaken and stretch with an increasing risk of dissection. For this reason an early and accurate diagnosis of the disease is essential [5]. For most people, MFS is not diagnosed until later in childhood or in adulthood. The MFS phenotype is evolutive, and the peculiar somatic traits of the syndrome are often the first signs suggestive of a diagnosis [6].

\footnotetext{
* chiarella.sforza@unimi.it; +39 02503 15387; www.scibis.unimi.it
} 
With particular reference to the craniofacial district, the clinical manifestations of MFS may include dolichocephaly, enophtalmos, palpebral downslanting, malar hypoplasia and retrognatia. These characteristics are comprised among the criteria applied for the diagnosis of MFS and they are usually evaluated through a clinical inspection or cranial X-ray examination [7-9]. Subjects with MFS may also have narrow jaws, high-arched palate, which can create dental and orthodontic problems such as teeth crowding, posterior crossbite and malocclusion [10-15). Sponseller et al. included craniofacial features of MFS among highly specific signs that musculoskeletal clinicians should be aware to improve the clinical recognition of the syndrome [16].

Today the two-dimensional assessment of craniofacial features on photographs and radiographic projections can be improved by means of the application of modern three-dimensional image acquisition systems which provide a detailed reconstruction of anatomic structures. Three-dimensional images are becoming a daily reality in several clinical and research contexts all over the world. Both volumetric (CT, MR) and surface (optical) imaging systems are currently being applied for the qualitative and quantitative assessments of craniofacial structures. In particular, instruments allowing a not invasive assessment of facial morphology such as stereophotogrammetry are of special interest, as they significantly improve the cost-benefit ratio of clinical analyses [17,18].

The three-dimensional morphometric facial analysis through stereophotogrammetry may highlight alterations of facial parameters which may be not detected by a clinical examination based on the mere inspection of the patients, and therefore give a contribution to the early diagnosis of the disease when the facial phenotype of MFS is not clearly evident or has not yet been clearly expressed. This is the key task for a correct strategy in preventing complications.

For instance, Kohler et al. hypothesized that the high occurrence of craniofacial dysmorphisms and an increased upper airway collapsibility could predispose to an increased prevalence of obstructive sleep apnoea (OSA) in MFS. Moreover, they demonstrated an association between OSA and aortic dilatation in MFS [19]. On the basis of clinical assessment of the orofacial phenotype of MFS, a recent study highlighted a correlation between a group of oral defects and systemic alterations of the syndrome, such as an aortic dilatation [9].

We aim to better describe the facial characteristics of patients with MFS, identifying new common quantitative morphological features which could facilitate the early diagnosis of the disease, in order to prevent the most serious complications of the syndrome. In the current preliminary study, a group of young Italian subjects with MFS was investigated.

\section{Method}

\subsection{Patients and control subjects}

Three girls and eight boys, fulfilling the Ghent criteria for MFS referred by the Rare Diseases Center MarfanClinic - Milan, were recruited for the study. We also examined 556 healthy subjects from the local population, matched with patients for gender, age, and ethnicity, to serve as controls. Participants were chosen among Italian Caucasoid subjects without history of facial surgical treatment or trauma. Details of the analyzed subjects are shown in Table 1.

Parents or legal guardians of involved subjects signed the written consent to the participation in the study, after explanation of its nature; verbal agreement was also provided from subjects themselves. All procedures were not invasive and not dangerous, were performed according to the tenets of the Declaration of Helsinki, and were preventively approved by the local ethic committee.

Table 1. Subjects analyzed in the current study.

\begin{tabular}{cccc}
\hline Age $(\mathbf{y r})$ & Gender & MFS & Controls \\
\hline $4-5$ & male & 2 & 98 \\
$8-11$ & male & 4 & 131 \\
$12-15$ & male & 2 & 195 \\
$12-15$ & female & 3 & 132 \\
\hline
\end{tabular}

\subsection{Data collection and analysis}

For each subject, the facial image was acquired using a three-dimensional stereophotogrammetric system (VECTRA M3, Canfield Scientific Inc, Fairfield, NJ, USA). The instrument has a $1.2 \mathrm{~mm}$ geometry resolution and can reproduce three-dimensional facial morphology with a capture time of 3.5 milliseconds.

Before each acquisition, 50 soft-tissue facial anthropometric landmarks were identified through inspection or palpation by an experienced examiner (Figure 1), according to international criteria and a 
specific experimental protocol developed and widely used by our laboratory in investigating facial dysmorphism also associated to genetic syndromes [20-27]. Each landmark was highlighted by a common commercial eyeliner, whose possible hypersensibility or intolerance were ascertained. The whole procedure took about 20 minutes.

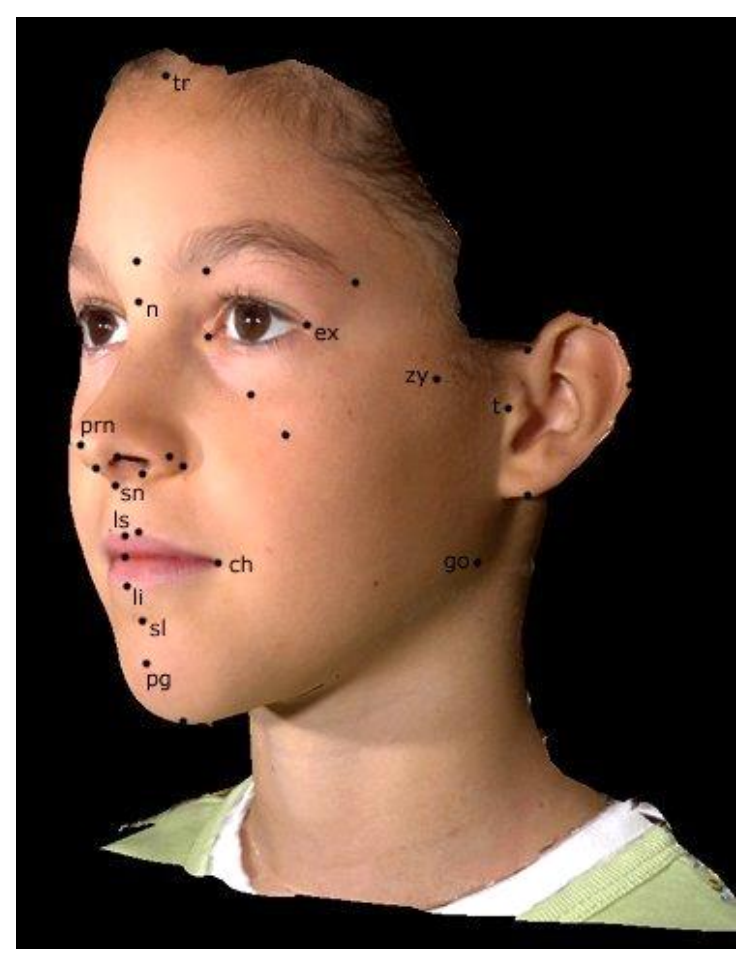

Fig. 1. Landmarks identified and digitized on all the subjects.

Landmarks used in the current study are associated with their names.

Using dedicated software, three-dimensional facial reconstructions were obtained. Data elaboration was performed off-line and was based on the three-dimensional coordinates of the digitized anthropometric landmarks, which allowed to obtain a geometric model of the face. Among the standard 50 landmarks, a subset of 8 selected midline landmarks and 5 paired landmarks descripted in Table 2 were considered in the current study.

Table 2. Facial landmarks used in the current study and relevant definitions.

\begin{tabular}{|c|c|c|}
\hline \multicolumn{3}{|c|}{ Midline landmarks } \\
\hline $\operatorname{tr}$ & trichion & on the hairline in the middle of the forehead \\
\hline $\mathrm{n}$ & nasion & at the suture between forehead and nose \\
\hline prn & pronasale & the most protruded point of the nasal apex \\
\hline sn & subnasale & at the end of the columella \\
\hline Is & labiale superius & midpoint of the vermilion line of the upper lip \\
\hline li & labiale inferior & midpoint of the vermilion line of the lower lip \\
\hline sl & sublabiale & in the midline of the mentolabial sulcus \\
\hline $\mathrm{pg}$ & pogonion & most anterior point of the chin \\
\hline \multicolumn{3}{|c|}{ Paired landmarks } \\
\hline ex & exocanthion & external commissura of the eye fissure \\
\hline zy & zygion & most lateral point of the zygomatic arch \\
\hline $\mathrm{t}$ & tragion & in the middle of the tragus \\
\hline ch & cheilion & outer labial commissura \\
\hline go & gonion & most lateral point of the mandibular angle \\
\hline
\end{tabular}

For each subject 13 facial linear distances and 15 facial angles were calculated by custom computer programs. Table 3 shows the performed measurements. 
Table 3. Analyzed distances and angles ( $r=$ right, $I=$ left, $m=$ mid-landmark).

\begin{tabular}{|c|c|}
\hline Linear distances & \\
\hline $\begin{array}{l}\text { Vertical distances } \\
\text { tr-n } \\
\text { n-sn } \\
\text { sn-pg }\end{array}$ & $\begin{array}{l}\text { length of the upper third of the face } \\
\text { length of the middle third of the face } \\
\text { length of the lower third of the face }\end{array}$ \\
\hline $\begin{array}{l}\text { Horizontal distances } \\
\text { ex }- \text {-ex } \\
z_{\mathrm{r}}-\mathrm{z}_{\mathrm{l}} \\
\mathrm{t}_{\mathrm{r}}-\mathrm{t}_{\mathrm{l}} \\
\mathrm{ch}_{\mathrm{r}}-\mathrm{ch}_{\mathrm{l}} \\
\mathrm{go}_{\mathrm{r}}-\mathrm{go}_{\mathrm{l}}\end{array}$ & $\begin{array}{l}\text { biocular width } \\
\text { face width } \\
\text { skull base width } \\
\text { mouth width } \\
\text { mandibular width }\end{array}$ \\
\hline $\begin{array}{l}\text { Sagittal distances } \\
t_{m}-n \\
t_{m}-s n \\
t_{m}-p g \\
\text { pg-go }_{m} \\
t_{m}-g_{m}\end{array}$ & $\begin{array}{l}\text { upper facial depth } \\
\text { mid facial depth } \\
\text { lower facial depth } \\
\text { mandibular body length } \\
\text { mandibular ramus length }\end{array}$ \\
\hline Angles & \\
\hline $\begin{array}{l}\text { Angles in the sagittal plane } \\
n-s n-p g \\
n-p r n-p g \\
\text { sn-n-prn } \\
\text { sl-n-sn } \\
\text { tr }_{r}-\mathrm{go}_{\mathrm{r}}-\mathrm{pg}, \mathrm{t}_{\mathrm{l}}-\mathrm{go}-\mathrm{pg} \\
\left(\mathrm{t}_{\mathrm{m}}-\mathrm{n}\right)-\left(\mathrm{go}_{\mathrm{m}}-\mathrm{pg}\right) \\
\text { prn-sn-ls } \\
\text { li-sl-pg } \\
\text { (sn-ls)-(li-pg) }\end{array}$ & $\begin{array}{l}\text { facial convexity, except nose } \\
\text { facial convexity, including nose } \\
\text { nasal convexity } \\
\text { maxillary prominence } \\
\text { mandibular angles (right and left) } \\
\text { facial divergence (midfacial to mandibular plane angle) } \\
\text { nasolabial angle } \\
\text { mentolabial angle } \\
\text { interlabial angle }\end{array}$ \\
\hline $\begin{array}{l}\text { Angles in the horizontal plane } \\
t_{r}-n-t_{1} \\
t_{r}-p r n-t_{1} \\
t_{r}-p g-t_{l} \\
g_{r}-p g-g o_{l} \\
\text { ex }-n-e x_{1}\end{array}$ & $\begin{array}{l}\text { upper facial convexity } \\
\text { middle facial convexity } \\
\text { lower facial convexity } \\
\text { mandibular convexity } \\
\text { relative position of exocanthia and nasion }\end{array}$ \\
\hline
\end{tabular}

Each measurement was expressed as $\mathrm{z}$ score value, calculated through the comparison with corresponding data obtained from control subjects (patient value minus mean value in the control subjects divided by the SD of the control subjects). The average $z$ scores and their standard deviations were then calculated. Since by definition the average $z$ score of control groups is $=0$ and its standard deviation is $=1$, we considered remarkable those mean patient $z$ scores that differed at least $1.4 \mathrm{SD}$ from the control values.

\section{Results}

Figure 2 shows examples of geometrical reconstruction of the face, starting from the three-dimensional coordinates of anatomical landmarks. The face of a 10-year-old boy with MFS and the average face of the group of healthy control subjects of the same age and sex are compared.

\subsection{Linear distances}

All subjects with MFS showed a longer face than controls, mainly due to an increase of the middle third (mean $z$ score $=1.7, S D=0.7$ ). Also the lower third of the face was longer (mean $z$ score $=1.6, S D=$ $0.4)$, while the upper third was not different. 
In comparison with control subjects, MFS patients showed different mandibular dimensions. In particular, a longer mandibular body was observed (mean $z$ score $=1.4, \mathrm{SD}=0.4$ ), while the mandibular ramus length was reduced (mean z score $=-1.4, \mathrm{SD}=0.9$ ). Upper, mid and lower facial depths were similar in subjects with MFS and controls.

All analyzed facial dimensions in the horizontal plane did not show apparent differences between MFS patients and control subjects.

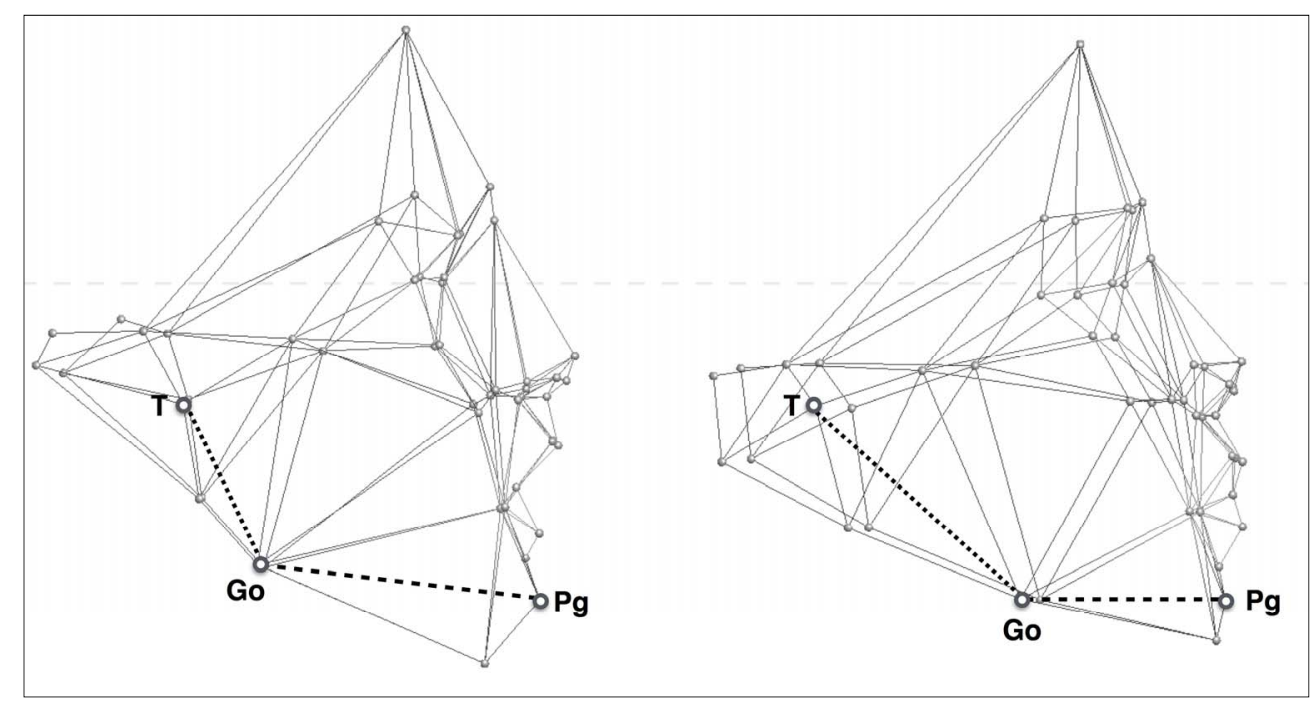

Fig. 2. Examples of 3D geometrical reconstruction of the face. The highlighted pogonion (pg)-gonion (go) and trago (t)-gonion (go) distances correspond to the mandibular body and ramus respectively.

Left: patient with MFS. Right: mean facial profile of 30 control subjects. All subjects are, male, 10 years old.

\subsection{Angles}

In the sagittal plane, all subjects with MFS showed a greater facial divergence (midfacial to mandibular plane angle) when compared to controls (mean $z$ score $=2.2, \mathrm{SD}=0.7$ ).

MFS patients did not show any remarkable differences in all the other analyzed angles in the three spatial dimensions. Nonetheless, a reduced upper facial convexity (mean z score $=-1.2, S D=0.7$ ) and an increased lower facial convexity (mean z score $=1.1, S D=0.8$ ) in the horizontal plane were pointed out.

\section{Discussion}

People born with MFS may not notice any features until later childhood or adulthood, but actually an early diagnosis of the syndrome is essential to prevent its life-threatening complications. Today, the evaluation of facial dysmorphism associated with MFS remains largely descriptive and almost based on subjective clinical impressions of the craniofacial features.

A recent study by Ting et al. analyzed the diagnostic value of facial features for MFS, concluding that the recognition of a specific facial phenotype can be used as an initial screening test. However, the study performed only a qualitative evaluation of two-dimensional photographs, in frontal and lateral views [28]. Anthropometry allows for a safe and non-invasive detailed qualitative and quantitative assessment of facial morphology and could help in the early diagnosis of the syndrome in clinical practice.

Beyond conventional direct anthropometry, which needs the direct contact with the subjects and exposes to the deformation of soft tissues, several procedures are now available for capturing and quantifying human three-dimensional facial surface morphology [29]. These include stereophotogrammetric systems, which are among the most appropriate for the definition of morphometric facial phenotypes, both in healthy subjects and in patients with different syndromes $[17,18]$. The high level of precision and repeatability of these systems is verified [30] and, due to the very short time required for the acquisition of the facial image, are appropriate for three-dimensional data collection even in children or disabled persons $[17,18]$. Moreover, they are easy to use and with practically no running costs.

Marking the landmarks of interest on the skin before the acquisition allows for capturing the position of those landmarks that can be efficaciously identified only by palpation of the underlying bone surface, thus increasing the instrument precision [18]. 
In the current preliminary study we evaluated the three-dimensional facial features of a small group of young subjects with MFS. Since the MFS phenotype is evolutive, the identification of specific facial features even in juvenile patients strengthens their usefulness for an early diagnosis of the syndrome. Facial linear distances and angles of subjects with MFS were compared to those obtained in healthy control subjects using $z$ scores. $Z$ scores values are well suitable for morphometric analyses, and they are widely used in clinical anthropometry to assess single patients $[8,23,25]$.

Some of the current findings are in good agreement with literature reports: the increased facial divergence can well explain the retrognatia listed among the typical facial features of subjects with MFS [7-9], even if we did not find actually reduced facial dimensions in the anteroposterior direction. Indeed, maxillo-mandibular reciprocal positions were found to be very variable in previous cephalometric studies [8], without significant modifications of the ANB angle [7], the skeletal equivalent of the current maxillary prominence angle. An actual increment in facial divergence was also reported by both Cistulli et al. [7] and De Coster et al. [8].

A longer face was previously reported for both Belgian and Australian subjects with MFS $[7,8]$. The increased palatal height found in English MFS subjects can also contribute to this vertical increment [19].

The present finding of an increased mandibular body length contrasts with previous cephalometric data, where the opposite result was reported [8]. Indeed, the use of two-dimensional radiographic projections, and the different age range of the analyzed subjects could partly explain the difference, even if further investigations are necessary. Additionally, no modifications in mandibular ramus length were previously listed $[7,8]$.

It has to be mentioned that the current investigation assessed facial soft tissues only, and no evaluations of the hard tissues were made. In contrast, previous studies used cephalometric analyses of head $x$ rays $[7,9,19]$. Additionally, we analyzed children and adolescent patients only, and compared their data with those of normal subjects of the same age, sex and ethnicity, while literature mainly reports data about adults $[7,19]$ or used reference values collected in other countries [8].

The quantitative assessment of facial features of subjects with MFS pointed out some morphometric characteristics that had never been reported in literature, alongside with other well known alterations: the current group of MFS patients showed a longer face due to an increase of its middle and lower thirds, a mandible with a longer body and a shorter ramus, and a greater facial divergence. These preliminary findings suggest the usefulness of a three-dimensional quantitative approach for the recognition of facial phenotypic features of MFS and encourage the evaluation of further anthropometric facial features. Nevertheless, they need to be confirmed extending the study on more patients.

\section{References}

[1] OMIM, http://omim.org/entry/154700, accessed on 14 June 2015.

[2] F. Ramirez and HC. Dietz, "Marfan syndrome: from molecular pathogenesis to clinical treatment", in Current Opinion in Genetics \& Development, Vol. 17, 2007, pp. 253-288, http://dx.doi.org/10.1016/i.gde.2007.04.006.

[3] A. Li-Wan-Po et al., "Preventing the aortic complications of Marfan syndrome: a case-example of translational genomic medicine", in British Journal of Clinical Pharmacology, Vol. 72, 2011, pp. 6-17, http://dx.doi.org/10.1111/i.1365-2125.2011.03929.x.

[4] BL. Loeys et al., "The revised Ghent nosology for the Marfan syndrome", in Journal of Medical Genetics, Vol. 47, 2010, pp. 476-485, http://dx.doi.org/10.1136/img.2009.072785.

[5] RM. Radke and H. Baumgartner, "Diagnosis and treatment of Marfan syndrome: an update" in Heart, Vol. 100, 2014, pp. 1382-1391, http://dx.doi.org/10.1136/heartjnl-2013-304709.

[6] C. Stheneur et al., "Study of phenotype evolution during childhood in Marfan syndrome to improve clinical recognition", in Genetics in Medicine, Vol. 16, 2014, pp. 246-250, http://dx.doi.org/10.1038/gim.2013.123.

[7] PA. Cistulli et al., "Relationship between craniofacial abnormalities and sleep-disordered breathing in Marfan's syndrome", in Chest, Vol. 120, 2001, pp. 1455-1460.

[8] P. De Coster et al., "Craniofacial structure in Marfan syndrome: a cefalometric study", in American Journal of Medical Genetics Part A, Vol. 131, 2004, pp. 240-248, http://dx.doi.org/10.1002/ajmg.a.30393.

[9] R. Docimo et al., "Association between oro-facial defects and systemic alterations in children affected by Marfan syndrome", in Journal of Clinical and Diagnostic Research, Vol. 7, 2013, pp. 700-703, http://dx.doi.org/10.7860/JCDR/2013/5656.2885. 
[10] JE. Bilodeau, "Retreatment of a patient with Marfan syndrome and severe root resorption", in American Journal of Orthodontics and Dentofacial Orthopedics, Vol. 137, 2010, pp. 123-134, http://dx.doi.org/10.1016/i.ajodo.2007.05.029.

[11] P. De Coster et al., "Oral manifestations of patients with Marfan syndrome: a case-control study", in Oral Surgery Oral Medicine Oral Pathology, Vol. 93, 2002, pp. 564-572, http://dx.doi.org/10.1067/moe.2002.121430.

[12] RH. Khonsari et al., "Extreme oral manifestations in a Marfan-type syndrome", in International Journal of Oral \& Maxillofacial Surgery, Vol. 39, 2010, pp. 622-625, http://dx.doi.org/10.1016/.ijom.2010.01.006.

[13] T. Nishikawa et al., "Marfan's syndrome: Clinical manifestations in the oral-craniofacial area, biophysiological roles of fibrillins and elastic extracellular microfibers, and disease control of the fibrillin gene", in Journal of Oral and Maxillofacial Surgery, Medicine, and Pathology, Vol. 25, 2013, pp. 374-388, http://dx.doi.org/10.1016/i.ajoms.2013.02.014.

[14] A. Utreja and CA. Evans, "Marfan syndrome-an orthodontic perspective", in Angle Orthodontist, Vol. 79, 2009, pp. 394-400, http://dx.doi.org/10.2319/112707-558.1.

[15] L. Westling et al., "Craniofacial manifestations in the Marfan syndrome: palatal dimensions and a comparative cephalometric analysis", in Journal of Craniofacial Genetics and Developmental Biology, Vol. 18, 1998, pp. 211-218.

[16] PD. Sponseller et al., "Improving clinical recognition of Marfan syndrome", in The Journal of Bone \& Joint Surgery, Vol. 92, 2010, pp. 1868-1875, http://dx.doi.org/10.2106/JBJS.I.00892.

[17] K. Aldridge et al., "Precision and error of three-dimensional phenotypic measures acquired from 3dMD photogrammetric images", in American Journal of Medical Genetics Part A, Vol. 138, 2005, pp. 247-253, http://dx.doi.org/10.1002/ajmg.a.30959.

[18] C. Sforza et al., "Soft- and hard-tissue facial anthropometry in three dimensions: what's new", in Journal of Anthropological Sciences, Vol. 91, 2013, pp. 59-84, http://dx.doi.org/10.4436/JASS.91007.

[19] M. Kohler et al., "The prevalence of obstructive spleep apnoea and its association with aortic dilatation in Marfan's syndrome", in Thorax, Vol. 64, 2009, pp. 162-166, http://dx.doi.org/10.1136/thx.2008.102756.

[20] LG. Farkas (ed), Anthropometry of the head and neck, 2nd ed., Raven Press, New York, USA, 1994, pp. 427, http://dx.doi.org/10.1002/hed.2880170222.

[21] VF. Ferrario et al., "A three-dimensional study of sexual dimorphism in the human face", in International Journal of Adult Orthodontics \& Orthognathic Surgery, Vol. 9, 1994, pp. 303-310.

[22] VF. Ferrario et al.,"Growth and aging of facial soft tissues: a computerized three-dimensional mesh diagram analysis", in Clinical Anatomy, Vol. 16, 2003, pp. 420-433.

[23] C. Sforza et al., "Variation in facial soft tissues of Italian individuals with ectodermal dysplasia", in Cleft Palate Craniofacial Journal, Vol. 41, 2004, pp. 262-267, http://dx.doi.org/10.1597/03-033.1.

[24] C. Sforza et al., "Abnormal variations in the facial soft tissues of individuals with Down syndrome: Sudan versus Italy", in Cleft Palate Craniofacial Journal, 2014 Oct 2. [Epub ahead of print], http://dx.doi.org/10.1597/14-082.

[25] C. Sforza et al., "Soft tissue facial morphometry in subjects with Moebius syndrome", in European Journal of Oral Sciences, Vol. 117, 2009, pp. 695-703, http://dx.doi.org/10.1016/j.ijom.2004.10.005.

[26] C. Sforza et al., "Three-dimensional facial distances of Northern Sudanese persons from childhood to young adulthood", in Journal of Cranio-Maxillo-Facial Surgery, Vol. 42, 2014, pp. e318-e326, http://dx.doi.org/10.1016/j.jcms.2013.10.013.

[27] M. De Menezes et al., "Evaluation of a 3D stereophotogrammetric method to identify and measure the palatal surface area in children with unilateral cleft lip and palate", in Cleft Palate Craniofacial Journal, 2015 Mar 20 [Epub ahead of print], http://dx.doi.org/10.1597/14-076.

[28] BL. Ting et al., "The diagnostic value of facial features of Marfan syndrome", in Journal of Children's Orthopaedics, Vol. 4, 2010, pp. 545-551, http://dx.doi.org/10.1007/s11832-010-0295-1.

[29] C. Sforza et al., "Three dimensional facial morphometry: from anthropometry to digital morphology", in Handbook of Anthropometry, Physical Measures of Human Form in Health and Disease. VR. Preedy (Ed), Springer Science+Business Media, LLC; Ch. 32, pp. 611-624, 2012; http://dx.doi.org/10.1007/978-1-4419-1788-1 32.

[30] M. de Menezes et al, "Accuracy and reproducibility of a 3-dimensional stereophotogrammetric imaging system", in Journal of Oral and Maxillofacial Surgery, Vol. 68, 2010, pp. 2129-2135, http://dx.doi.org/10.1016/.joms.2009.09.036. 